\title{
Etiquetado nutricional frontal y su impacto en la salud pública. Consideraciones bioéticas
}

\author{
Nutritional front labeling and its impact on public health. Bioethical \\ considerations \\ Rotulagem nutricional frontal e seu impacto na saúde pública. Considerações boéticas
}

\author{
Lina María Sierra Tobón ${ }^{1 *}$
}

Recibido: 30 de septiembre de 2020. Aceptado para publicación: 01 de abril de 2021.

Publicado en línea: abril 2021.

https://doi.org/10.35454/rncm.v4n3.214

\section{Resumen}

Introducción: en los últimos años, el etiquetado nutricional frontal se ha recomendado como política de protección del derecho a la salud, con el argumento de que permite orientar al consumidor en la elección de alimentos saludables, incentivar a la industria en la reformulación de productos sanos y facilitar el diseño de políticas públicas de alimentación saludable, siendo uno de los principales motivos de su implementación el aumento en la prevalencia de enfermedades no transmisibles relacionadas con la dieta.

Objetivo: revisar el impacto del etiquetado nutricional frontal en la salud pública y proponer algunas consideraciones bioéticas que favorezcan su efectividad.

Métodos: se realizó una búsqueda bibliográfica, revisando el impacto de dicha medida sobre la salud pública, seguido de una reflexión bioética en torno al tema.

Resultados: si bien es cierto, el etiquetado frontal puede incidir en la elección de alimentos, la decisión sobre la compra y el consumo está influenciada por aspectos propios del individuo y su contexto. Se propone considerar el empoderamiento del consumidor, la corresponsabilidad de la industria alimentaria y la protección del estado como fundamentos bioéticos que, acompañados de la discusión en torno a la esencia de la libertad humana y el condicionamiento de los determinantes socia-

\section{Summary}

Introduction: Front-of-pack nutrition labeling has been recommended in recent years as a policy to protect the right to health, arguing that it allows to guide the consumer in the choice of healthy foods, encourage the industry in the reformulation of healthy products, and facilitate the design of public policies for healthy eating, one of the main reasons for its implementation being the increase in the prevalence of diet-related non-communicable diseases.

Objective: To review the impact of front-of-pack labeling on public health and to propose some bioethical considerations to further its effectiveness.

Methods: A literature search was carried out reviewing the impact of this measure on public health, followed by a bioethical reflection on the subject.

Results: Although it is true that frontof-pack labeling can influence food choice, purchase and consumption decisions are influenced by factors related to the individual and his or her context. Consumer empowerment, co-responsibility of the food industry and state protection are proposed as bioethical foundations that, accompanied by the discussion around the essence of human freedom and the conditioning of social determinants of health, would favor labelling effectiveness.

\section{Resumo}

Introdução: A rotulagem nutricional frontal tem sido recomendado nos últimos anos como política de proteção ao direito à saúde que permite orientar o consumidor na escolha de alimentos saudáveis, incentivar à indústria a reformular produtos saudáveis e viabilizar a formulação de políticas públicas de alimentação saudável, sendo um dos principais motivos para sua implantação o aumento da prevalência de doenças não transmissíveis relacionadas à alimentação.

Objetivo: Revisar o impacto da rotulagem nutricional frontal na saúde pública e propor algumas considerações bioéticas que favoreçam sua eficácia.

Métodos: Foi realizada uma pesquisa bibliográfica revisando o impacto dessa medida na saúde pública, seguida de uma reflexão bioética sobre o tema.

Resultados: Embora seja verdade que a rotulagem frontal pode influenciar a escolha de alimentos, a decisão de compra e consumo é influenciada por aspectos do indivíduo e de seu contexto. Propõe-se considerar o empoderamento do consumidor, a corresponsabilidade da indústria de alimentos e a proteção do estado, como fundamentos bioéticos que, acompanhados da discussão em torno da essência da liberdade humana e do condicionamento dos determinantes sociais da saúde favoreçam sua eficácia. 
les de salud, favorecerían su efectividad.

Conclusiones: la reflexión bioética en torno a la triada consumidor/industria/ estado permite fundamentar una buena praxis en la implementación del etiquetado nutricional frontal de alimentos como política de salud pública.

Palabras clave: etiquetado de alimentos, salud pública, bioética.
Conclusions: The bioethical reflection around the consumer, industry and state triad allows to base good praxis on frontof-pack food labelling implementation as a public health policy.

Keywords: Nutritional Labeling; Public Health; Bioethics.
Conclusões: A reflexão bioética em torno da tríade: consumidor, indústria, estado, permite justificar uma boa prática na implementação da rotulagem nutricional frontal de alimentos, como política de saúde pública.

Palavras-chave: rotulagem de alimentos, saúde pública, bioética
Nutrición y Dietética; Facultad de Medicina, Clínica Alemana Universidad del Desarrollo, Santiago de Chile, Chile.
*Correspondencia: Lina María Sierra Tobón. integralclinicalnutrition@gmail.com

\section{INTRODUCCIÓN}

De acuerdo con la Organización de las Naciones Unidas para la Alimentación y la Agricultura (FAO), el etiquetado de alimentos corresponde a la información presentada en los productos alimentarios, siendo uno de los medios más importantes y directos para transmitir la información al consumidor sobre los ingredientes, la calidad o el valor nutricional ${ }^{(1)}$. El etiquetado frontal hace referencia a la información que se presenta de manera gráfica en la cara principal del producto ${ }^{(2)}$. Éste tiene por objetivo ofrecer información veraz, simple y clara respecto al contenido nutricional de alimentos y bebidas no alcohólicas.

Dicho etiquetado se ha recomendado como política pública para la protección del derecho a la salud ${ }^{(3)}$, al argumentar que facilita el acceso a la información nutricional respecto de los paneles tradicionales y ayuda al consumidor a elegir alimentos calificados como saludables de acuerdo con un sistema de perfil de nutrientes. La Organización Panamericana de la Salud (OPS) lo plantea como una de sus líneas de acción para la prevención de la obesidad en la niñez y la adolescencia ${ }^{(4)}$. De igual manera, la Organización Mundial de la Salud (OMS) propone su puesta en marcha como apoyo a la educación del consumidor, para que tanto adultos como niños tengan conocimientos básicos en materia de nutrición ${ }^{(4)}$.

En la actualidad existen múltiples experiencias de etiquetado frontal ${ }^{(5)}$, la mayoría de carácter voluntario, siendo Latinoamérica una de las regiones más avanzadas en materia regulatoria. Dentro de las medidas más reconocidas se encuentran Semáforo (Ecuador, 2014), Advertencia (Chile, 2016), Semáforo (Bolivia, 2016),
Advertencia (Perú, 2018) y Advertencia (Uruguay, $2018)^{(6)}$. Otros países, como Argentina y Brasil, están en proceso de discusión sobre el proyecto de etiquetado frontal, mientras que México recientemente (octubre de 2020) adoptó el etiquetado frontal de advertencia.

Por su parte, el Ministerio de Salud y Protección Social de Colombia, como estrategia para la promoción de la salud pública, en febrero de 2020 dio a conocer la propuesta de etiquetado nutricional frontal, que deben cumplir los alimentos envasados, cuya obligatoriedad se estima comenzará en $2022^{(7)}$. Dicha medida tiene por objetivo presentar la información nutricional de forma clara y comprensible para el consumidor, al incluir el sello frontal de advertencia, con reporte de "alto en azúcares añadidos, sodio o grasas saturadas" y evolucionar el marco normativo al pasar de acciones voluntarias a obligatorias (Figura 1).

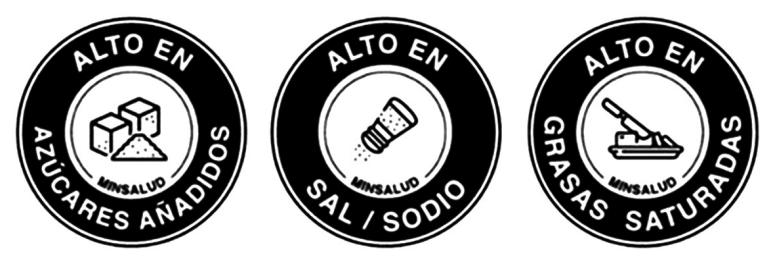

Figura 1. Rotulado de advertencia con reporte de alto en azúcares añadidos, sodio o grasas saturadas propuesto para Colombia $^{(7)}$.

A portas de la ejecución de la norma se revisa de manera preliminar, el impacto del etiquetado nutricional frontal sobre la salud pública, encontrando que si bien es cierto favorece la elección de alimentos, se debe ser cauteloso frente a su expectativa, teniendo en cuenta los diversos aspectos que inciden sobre la decisión de 
compra y consumo. Por tanto, el presente artículo tiene por objetivo revisar la literatura referente al impacto del etiquetado nutricional frontal sobre la salud pública, y discutir algunas consideraciones bioéticas que pueden favorecer su efectividad.

A partir de la revisión realizada se propone el empoderamiento del consumidor, la corresponsabilidad de la industria alimentaria y la protección del estado, como consideraciones bioéticas para tener en cuenta. Finalmente, se ofrece una breve discusión en torno a la esencia de la libertad humana y los determinantes sociales de salud (DSS), como aspectos que inciden en el proceso de selección, compra y consumo de alimentos.

Es así como la relevancia del presente artículo se da por la inminente necesidad de acompañar la implementación de medidas de salud pública de una reflexión bioética en torno al tema.

\section{MÉTODOS}

Se realizó una revisión bibliográfica donde se consultaron artículos académicos, utilizando como criterio de búsqueda [(tema contiene) "etiquetado frontal de alimentos" AND "salud pública", filtrando los artículos en inglés y español publicados durante los últimos 10 años (2010-2020), y seleccionando aquellos que se relacionaban de forma directa con el tema. Acto seguido se realizó una revisión en torno a la ética de la salud pública, con el objetivo de reconocer algunas consideraciones bioéticas que pudieran favorecer la efectividad del etiquetado nutricional frontal.

\section{RESULTADOS}

La revisión llevada a cabo permitió agrupar los hallazgos en las siguientes dos categorías:

\section{Impacto del etiquetado frontal en la salud pública}

Un reciente estudio ${ }^{(8)}$ sintetizó la información disponible sobre el efecto del etiquetado nutricional frontal en la elección, compra y consumo de alimentos y bebidas, y el estado nutricional de los consumidores, e identificó los factores que influyen en su efectividad. Los resultados señalan que la etiqueta frontal facilitó la elección correcta de alimentos saludables y tuvo un efecto variable sobre las dimensiones de consumo y compra, mientras que factores relacionados con el individuo y su entorno parecen tener una influencia importante en su efectividad.
Otras revisiones ${ }^{(9)}$ indican que hay mecanismos cognitivos que pueden influenciar la eficacia del etiquetado frontal, que van desde una simple mirada y confianza parcial, hasta un procesamiento profundo por parte del consumidor, aspecto que explicaría la variada efectividad de los distintos formatos, lo que incide en la selección del modelo más apropiado para cada país.

Actualmente no existe un sistema de etiquetado frontal que sea de aceptación general. La propuesta de algunos autores es que su diseño se haga basado en los nutrientes considerados como prioridad para la salud pública de cada país y que sea fácil de comprender por el consumidor ${ }^{(10)}$.

Al revisar los tipos de etiquetado frontal adoptados por países de la región, se aprecia que el sistema de advertencia propuesto por Chile, e implementado recientemente en México, es uno de los que ha demostrado mayor eficacia para informar a los consumidores, favorecer la elección de alimentos saludables, identificar los menos saludables e influenciar los patrones de compra ${ }^{(11-13)}$.

Teniendo en cuenta que su modelo es similar al que implementará Colombia, resulta pertinente dar una mirada a la experiencia de Chile como país pionero en el tema, liderazgo que se le ha atribuido gracias a la implementación de la ley 20.606 sobre composición nutricional de los alimentos y su publicidad ${ }^{(14)}$. Dicha ley incorpora un marco regulatorio que permite:

- Entregar información más clara y comprensible al consumidor por medio del sello de advertencia.

- Asegurar una oferta saludable de alimentos al interior de los establecimientos educativos.

- Proteger a los menores de 14 años de la sobreexposición a la publicidad.

La ley de etiquetado, como se le conoce, se ha considerado por organismos internacionales como ejemplo de estrategia para la rotulación frontal de alimentos. Esta se destaca como política regulatoria integral para hacer frente a la epidemia de la obesidad, dado que facilita el diseño e implementación de políticas públicas de alimentación saludable, e incentiva a la industria a reformular sus productos, haciéndolos más sanos.

Respecto a su impacto, el Instituto de Nutrición y Tecnología de los Alimentos (INTA) señala que esta iniciativa tiene el potencial de mejorar la comprensión nutricional de la población y modificar conductas de compra en algunas categorías de alimentos. Sin embargo, indica que para evaluar el efecto de su implementación sobre la salud pública se requiere de un mayor tiempo de exposición, que permita evidenciar cambios en la dieta y adiposidad de la población ${ }^{(15)}$. 
Lo anterior tiene explicación en el tiempo que toma la modificación de hábitos alimentarios relacionados con la efectividad del etiquetado ${ }^{(16)}$. De acuerdo con revisiones recientes, "decidir qué y cuánto comer es para muchos consumidores un comportamiento basado en hábitos"(8), no contando hasta ahora con suficiente evidencia que respalde el impacto del etiquetado sobre los hábitos alimentarios del consumidor.

La revisión sistemática más reciente ${ }^{(8)}$ señala que la evidencia científica concerniente al impacto del etiquetado nutricional frontal en la salud pública es limitada, teniendo en cuenta que su efecto en la morbimortalidad no es claramente demostrable antes de una década. Indica que, "los estudios observacionales con modelamiento estadístico consideran que el etiquetado frontal produciría una disminución significativa del peso, mejoras sustanciales en el consumo de nutrientes y una reducción moderada del consumo de energía y del riesgo cardiovascular"(8).

Se reconoce entonces que los factores individuales y contextuales que limitan la efectividad del etiquetado nutricional obliga a que su implementación en los países de Latinoamérica se acompañe de un marco de políticas más amplio, que incluya, por ejemplo, estrategias para mejorar el acceso a los alimentos saludables, ofrecer educación nutricional a los consumidores y promover la actividad física.

$\mathrm{Al}$ respecto, la $\mathrm{FAO}^{(1)}$ señala que para aprovechar al máximo el potencial de las etiquetas nutricionales y las declaraciones de propiedades saludables orientadas a mejorar la salud pública, es importante reconocer que el uso adecuado de las etiquetas requiere de campañas de sensibilización y programas educativos que ayuden a los consumidores a comprenderlas y utilizarlas. Esta primera revisión reconoce las limitaciones, pero, a la vez, el potencial que podría tener el etiquetado nutricional frontal como política de salud pública para combatir la prevalencia de enfermedades crónicas no transmisibles.

Dicho reconocimiento permite proponer algunas consideraciones bioéticas que favorezcan su efectividad, teniendo en cuenta que, si bien la etiqueta frontal puede influenciar la elección de alimentos, la decisión sobre compra y consumo está determinada por factores propios del individuo y su entorno.

\section{Consideraciones bioéticas que pueden favorecer la efectividad del etiquetado nutricional frontal}

La ética de la salud pública merece especial atención por ser el área de desarrollo más reciente de la bioética como disciplina, por la ubicuidad de la acción en salud pública y la consideración de los DSS. Se diferencia de la ética clínica esencialmente en su objeto central de análisis. A pesar de compartir la salud como categoría genérica, ambos contextos tienen finalidades diferentes ${ }^{(17)}$. Dicho de otra manera, la medicina permite prevenir y curar las enfermedades humanas, mientras que la salud pública corresponde a una actividad encaminada a mejorar la salud de la población, lo que origina finalidades diferentes y, por tanto, una ética asumida desde el objeto central de cada una de ellas.

Es así como la ética clínica plantea la coexistencia de cuatro principios: beneficencia, no maleficencia, autonomía y justicia, enunciados para la investigación en seres humanos y bioética médica. Estos lineamientos hacen referencia a interacciones entre individuos, como ocurre en la relación médico-paciente o en la de investigador-análisis ${ }^{(18)}$.

Dicho modelo principialista pareciera ser insuficiente para enfrentar los dilemas actuales de la salud pública ${ }^{(18)}$, considerando que aunque ambas se preocupan por el bienestar humano desde el punto de vista de las enfermedades, su tratamiento y su eventual curación, tanto la medicina como la salud pública tienen su propia especificidad teórica, práctica y normativa. Partiendo de su objeto de análisis, la ética médica se centra en la relación médico-paciente, mientras que la ética de la salud pública apunta al diseño y aplicación de medidas para la vigilancia y mejora de la salud de las poblaciones, lo que trasciende la atención sanitaria, para considerar las condiciones estructurales que promueven o dificultan el desarrollo de sociedades sanas ${ }^{(17)}$.

Lo descrito permite proponer, de manera preliminar, 3 consideraciones bioéticas que pueden contribuir a favorecer la efectividad del etiquetado nutricional frontal como política de salud pública, cuyo referente universal son las medidas colectivas (Figura 2):

- El empoderamiento del consumidor.

- La corresponsabilidad de la industria alimentaria.

- La protección del estado.

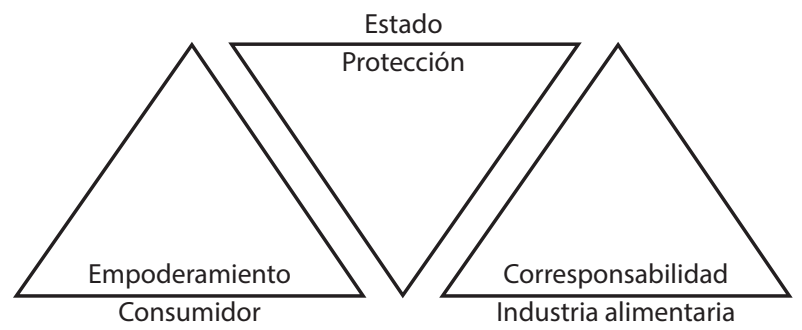

Figura 2. Consideraciones bioéticas propuestas: empoderamiento del consumidor, corresponsabilidad de la industria alimentaria y protección del estado. 


\section{Empoderamiento del consumidor}

La Declaración de Cartagena sobre el derecho al cuidado nutricional y la lucha contra la malnutrición ${ }^{(19)}$ hace referencia al "empoderamiento" como una acción necesaria para mejorar el cuidado nutricional. Lo define como un proceso basado en el principio de que al aumentar la educación se mejora la capacidad de pensar en forma crítica y de actuar de manera autónoma, con un resultado alcanzado mediante el sentido de autoeficacia, correspondiendo a la parte final del proceso.

En el campo clínico, el empoderamiento busca aumentar la libertad y autonomía del paciente capacitándolo en la toma de decisiones informadas. En el caso de la salud pública, el reto del empoderamiento radica en educar a la sociedad para que de una manera libre y responsable pueda elegir, por ejemplo, entre un alimento $\mathrm{u}$ otro. $\mathrm{Al}$ respecto, los principios del Codex establecen que el etiquetado nutricional debe ser un medio para facilitar la información al consumidor, de manera tal que éste pueda elegir su alimentación con discernimiento ${ }^{(20)}$.

Algunos autores ${ }^{(21)}$ indican que el acceso del consumidor a una información veraz y confiable respecto al alimento que compra es un derecho ineludible, y que, por tanto, la mejor forma de presentarla es en la parte frontal del empaque. En ese sentido, el Observatorio de Bioética y Derecho (OBD) de la Universidad de Barcelona menciona que "la tesis fundamental de los poderes públicos es que la información alimentaria permite garantizar la libertad del consumidor para realizar elecciones informadas, así, si el ciudadano consume alimentos poco saludables, ello ocurre en el contexto de una elección individual de la que él es responsable, la etiqueta le informó e, incluso así, decidió consumir el alimento"(22).

El mencionado observatorio advierte que organizaciones internacionales, como la OMS y la FAO, han manifestado que las elecciones alimentarias no responden tan solo a condicionantes relativos a la información disponible, refiriéndose a la función limitada del etiquetado, siendo insuficiente, entre otras cosas, porque junto a los factores estrictamente nutricionales o sanitarios hay que tener en cuenta otros elementos determinantes en la toma de decisiones.

En suma, la información descrita en el etiquetado frontal busca empoderar al consumidor, sin embargo, frente a esta primera consideración bioética debe tenerse en cuenta que los hábitos alimentarios no siempre se eligen de manera libre, al estar relacionados con condiciones socioeconómicas, culturales y de marketing, lo que hace presumir la importancia de las siguientes dos consideraciones: corresponsabilidad de la industria alimentaria y protección del estado.

\section{Corresponsabilidad de la industria alimentaria}

La corresponsabilidad, definida como responsabilidad compartida, corresponde a un valor social insustituible para lograr alcanzar los objetivos de un estado social de derecho. Ésta implica que la responsabilidad no es exclusiva del ente estatal, sino de todos sus miembros ${ }^{(23)}$.

Entender que la responsabilidad es compartida conlleva a tener una actitud proactiva, desde el autocuidado al que se puede llegar a través del empoderamiento, hasta el deber del estado de intervenir y dar las herramientas suficientes para que los ciudadanos obtengan la protección de sus derechos a través de políticas públicas efectivas.

La noción de corresponsabilidad se fundamenta en una concepción de democracia participativa, en la que la gestión pública involucra a la sociedad civil ${ }^{(24)}$, incluyendo, por ejemplo, a la industria de alimentos en el diseño y el cumplimiento de recomendaciones que son de interés general.

De acuerdo con la $\mathrm{OBD}^{(22)}$, el principio de corresponsabilidad obliga a la industria a velar porque sus productos cumplan los requisitos de la legislación alimentaria en todas las etapas de producción, transformación y distribución, que garanticen no solo la seguridad, sino la calidad de los alimentos, e insta a los productores a respetar las expectativas de los ciudadanos respecto de la calidad del producto, cuyas características deben ser apreciables a través del etiquetado nutricional.

De igual manera, el etiquetado frontal, en cualquiera de sus sistemas, representa un incentivo para que los productores de alimentos, los representantes de Salud Pública y las Agencias Reguladoras fomenten tanto la reformulación de los productos, como la creación de estrategias de marketing y la unificación del etiquetado de alimentos y bebidas no alcohólicas ${ }^{(21)}$. Lo anterior, teniendo en cuenta que con el aumento del comercio globalizado y la desaparición del modelo tradicional de relación frente a frente entre los productores de alimentos y consumidores, existe una mayor necesidad de crear etiquetas alimentarias que sean confiables y que no resulten engañosas ${ }^{(25)}$.

Esta segunda consideración bioética reconoce el desafío que la industria de alimentos procesados tiene, a fin de garantizar la calidad de sus alimentos, elegir mejores ingredientes y plantear estrategia de mercadeo que orienten la sana elección, compra y consumo, ade- 
más de garantizar precios justos, entre otros aspectos, que permitan el acceso a una sana alimentación. Por lo anterior, la corresponsabilidad de la industria alimentaria y el desafío que ello conlleva debe ir acompañado de la vigilancia del ente estatal, aspecto en el que se fundamenta la protección del estado como tercera consideración bioética propuesta.

\section{Protección del estado}

En salud pública, la protección se entiende como la actitud de dar resguardo o cobertura a las necesidades esenciales, es decir, aquellas que deben ser satisfechas para que el afectado pueda atender a otras necesidades u otros intereses. La ética de la protección sanitaria es vista como una propuesta de cuidar a la ciudadanía, con miras a prevenir enfermedades y fomentar un medio ambiente saludable. Ésta no se confunde con paternalismo, dado que el agente protector no puede actuar sin el consentimiento de la población, por lo que se deben proponer medidas públicas necesarias y razonables para prevenir los problemas sanitarios ${ }^{(18)}$.

El principio de protección ofrece la posibilidad de una evaluación ética de acciones destinadas a cubrir necesidades sanitarias impostergables, efectivamente sentidas por la población ${ }^{(18)}$. La legitimidad de las acciones sanitarias, y la inevitable restricción de la autonomía individual, constituyen características de los actos protectores. Al respecto, el Grupo de Opinión del $\mathrm{OBD}^{(22)}$ resalta que la falta de garantías efectivas en la aplicación de las leyes lleva a la desconfianza por parte del consumidor, tanto por actuación de la industria, como por la capacidad de los poderes públicos para salvaguardar sus derechos, lo que evidencia la importancia de esta tercera consideración, donde se reconoce al estado como ente protector y garante.

Finalmente, las consideraciones bioéticas propuestas (empoderamiento del consumidor, corresponsabilidad de la industria alimentaria y protección del estado) representan un punto de partida sobre el cual apoyar la reflexión bioética en torno a la efectividad del etiquetado nutricional frontal como política de salud pública.

\section{ANÁLISIS Y DISCUSIÓN DESDE UN ENFOQUE ÉTICO}

Una de las personas que conoce con mayor precisión la ley de etiquetado nutricional en Chile, es Samuel Durán Agüero, quien es académico investigador de la Universidad San Sebastián y director del Magíster de
Nutrición en Salud Pública. Al ser interrogado respecto al impacto de la ley de etiquetado nutricional señala que: "La gente sabe lo que es bueno o malo comer, pero no está motivada a elegir alimentos sin sellos"(26).

Lo descrito lleva a pensar que las consideraciones bioéticas mencionadas (empoderamiento, corresponsabilidad y protección) deben acompañarse de la discusión en torno a la esencia de la libertad humana y los determinantes sociales en salud, aspectos que inciden en el proceso de selección, compra y consumo de alimentos y que, por tanto, influyen sobre la efectividad del etiquetado nutricional como política de salud pública.

\section{ESENCIA DE LA LIBERTAD HUMANA}

La autodeterminación hace referencia a la capacidad individual y colectiva para decidir por sí mismo. De acuerdo con Kant, hay un sentido más amplio de autodeterminación y, por tanto, de libertad práctica. Se trata de la capacidad de determinarse no por instinto, como los demás animales, sino por medio de representaciones intelectuales ${ }^{(27)}$.

Para el filósofo alemán, el ser humano no funciona al modo estímulo-respuesta, sino que está en su poder configurar racionalmente la respuesta a los diversos estímulos. La libertad corresponde a la capacidad de los seres racionales para determinarse a obrar según leyes distintas a las naturales, ésto es según leyes que son dadas por su propia razón. Libertad equivale entonces a la autonomía de la voluntad ${ }^{(28)}$.

En el mismo sentido, Burgos ${ }^{(29)}$ resume la estructura esencial de la libertad en la expresión: "yo quiero algo", es un "algo" que al ser elegido repercute sobre el "yo" y lo modifica; nos modificamos a nosotros mismos cuando ejercemos la acción de "querer". Para el tema en cuestión ¿Qué alimento quiero? y ¿Cómo esa elección me modifica?

De acuerdo con el autor, la libertad consta de elección (la libertad como independencia de los objetos) y autodeterminación (la libertad como dependencia de sí), refiriéndose al problema del determinismo, donde se postula una primacía del objeto sobre el hombre y reconoce el casi irresistible como el punto donde se juega la esencia de la libertad. Para Burgos "la acción voluntaria y libre procede de la persona”.

$\mathrm{Al}$ analizar el tema a la luz de lo discutido, nos encontramos frente a políticas públicas que, como parte de la normativa, impiden publicitar alimentos considerados poco saludables, dejar de ofrecerlos en establecimientos educativos y etiquetarlos con sellos de advertencia, 
pareciendo que fuera el objeto quien decide sobre el individuo y no el individuo sobre el objeto.

Sin dejar de reconocer la efectividad de dichas políticas, el mayor esfuerzo debe ir orientado a educar a las personas sobre el riesgo que implica para su salud abusar del consumo de alimentos "altos en", y no solo poner el énfasis en prohibirlos, etiquetarlos o no publicitarlos, dándole la oportunidad de decidir de manera libre y voluntaria si lo consume o no. De esta manera, hablaríamos de una elección intencional, independiente del objeto, con procedencia causal, haciendo al hombre responsable de sus acciones, motivándolo a elegir alimentos sin sellos.

Sin embargo, la evidencia ha demostrado que la elección de alimentos está condicionada no solo por la esencia de la libertad, de ser así bastaría con empoderar al consumidor para que de manera libre elija el alimento más conveniente para su salud, sino por determinantes sociales que condicionan dicha elección y que son ineludibles cuando se discute temas relacionados con la bioética de la salud pública.

\section{LOS DETERMINANTES SOCIALES DE SALUD}

Los DSS están definidos por la $\mathrm{OMS}^{(30)}$ como las circunstancias en que las personas nacen, crecen, trabajan, viven y envejecen, incluido el sistema de salud. Dicho de otra manera, las características sociales en las que la vida se desarrolla, y que, para el caso, suelen condicionar la decisión de compra y consumo. El modelo de Dahlgren y Whitehead (Figura 3) explica cómo las inequidades en salud son resultado de las interacciones entre distintos niveles de condiciones causales, desde el individuo hasta las comunidades ${ }^{(31)}$.

Los DSS representan, por tanto, una valiosa oportunidad para que los gobiernos, a través de sus políticas públicas, logren disminuir la brecha social, llegando al punto donde la decisión de compra para todos los integrantes de la comunidad dependa de la elección libre y responsable.

Para actuar respecto a los determinantes sociales es necesario reconocer las causas complejas, y a menudo duraderas, de las enfermedades crónicas no transmisibles, y abordar las "causas de las causas" asociadas con la inequidad en materia de salud mediante la investigación desde las ciencias sociales y la epidemiología, lo que puede eliminar o minimizar algunos de los principales obstáculos que repercuten en la salud individual, y apoyar la transición progresiva hacia la salud universal.

En ese sentido, Bourdieu hace referencia al habitus como el conjunto de esquemas generativos, a partir de los cuales los sujetos perciben el mundo y actúan en él. Para el sociólogo francés, dicho concepto permite

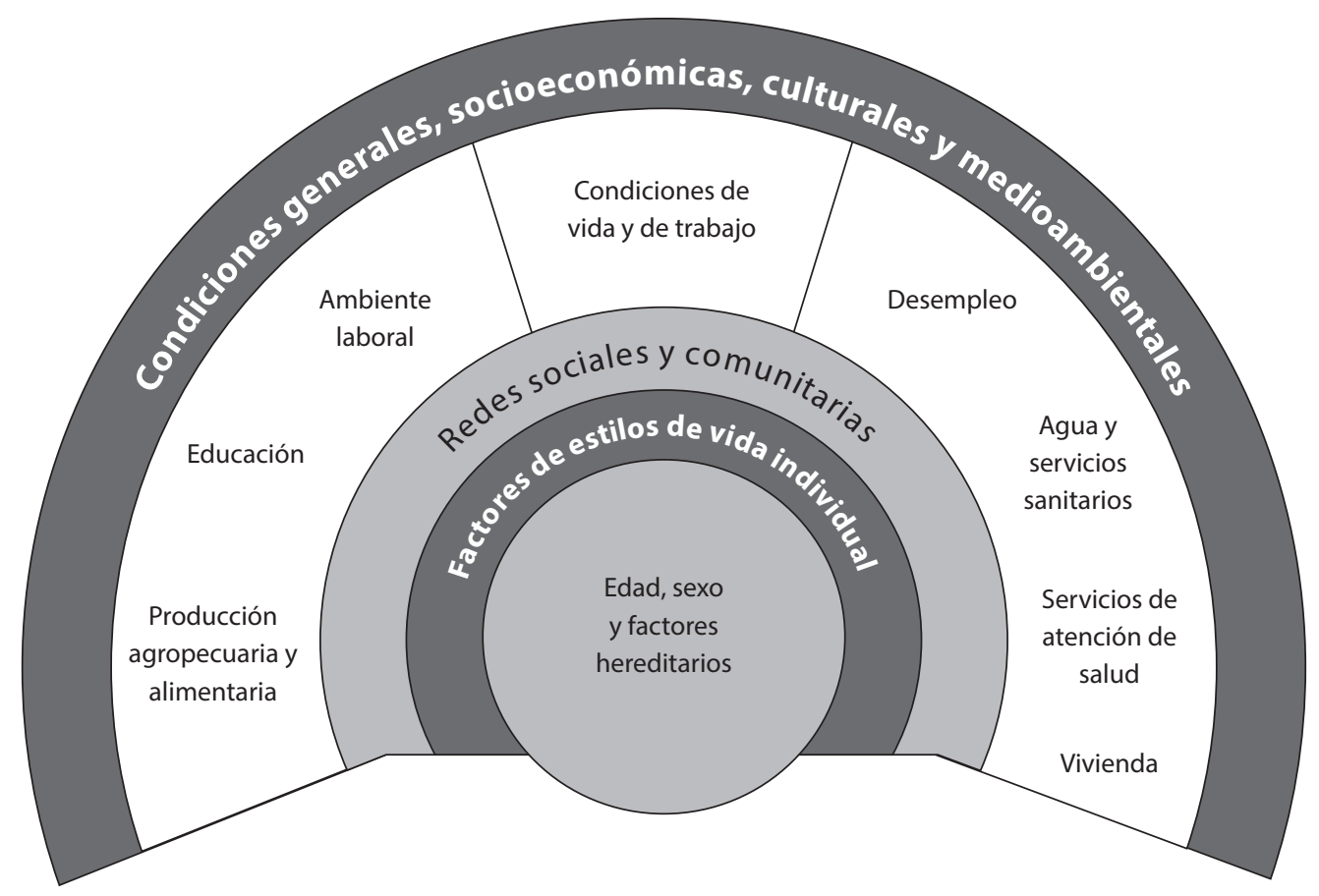

Figura 3. Modelo de Dahlgren y Whitehead sobre determinantes de la salud. Adaptada de ${ }^{(31)}$. 
entender "disposiciones" o esquemas de obrar, pensar y sentir asociados con la posición social ${ }^{(32)}$.

De acuerdo con este concepto podemos reconocer habitus comunes entre los habitantes de una determinada región, o entre un grupo de la misma edad, o entre quienes tienen similar nivel de ingresos. Dicho habitus condiciona de alguna manera la decisión de compra y, por tanto, la efectividad de las políticas de salud pública, como lo es el etiquetado nutricional. Por lo anterior se recomienda que la elección del etiquetado nutricional frontal se haga pensando en el habitus que condiciona la libertad del consumidor y que el mayor esfuerzo del estado, como ente protector, vaya orientado a disminuir las inequidades existentes, teniendo en cuenta los DSS, e involucrando al consumidor y a la industria de alimentos en la implementación de políticas públicas efectivas.

\section{CONCLUSIÓN}

El etiquetado nutricional frontal puede incidir favorablemente en la elección de alimentos, sin embargo, se debe ser cauteloso frente a la expectativa, teniendo en cuenta que la decisión de compra y consumo está influenciada por aspectos propios del individuo y su contexto.

La limitación de esta revisión se da por el poco tiempo transcurrido desde la implementación del etiquetado nutricional frontal en los diferentes países de Latinoamérica, lo que resulta ser insuficiente para determinar el real impacto de dicha medida sobre la prevalencia de enfermedades crónicas no trasmisibles.

La revisión realizada propone, de manera preliminar, considerar el empoderamiento del consumidor, la corresponsabilidad de la industria alimentaria y la protección del estado como principios que podrían favorecer la efectividad del etiquetado nutricional frontal como política de salud pública.

La reflexión bioética permitió reconocer la esencia de la libertad humana y la manera como es condicionada por los DSS, aspecto que, unido al habitus, incidiría en el éxito del etiquetado nutricional frontal como política de salud pública.

El artículo presentado abre la puerta a futuras revisiones que permitan documentar el real impacto del etiquetado nutricional frontal sobre la prevalencia de enfermedades crónicas no trasmisibles, cuyos resultados permitan profundizar en consideraciones bioéticas que favorezcan su efectividad.

\section{Agradecimiento}

Agradezco a la Asociación Colombiana de Nutrición Clínica la constante motivación para que el profesional de salud se interese en investigar, proponer y publicar artículos de calidad científica que enriquezcan el ethos profesional.

\section{Financiamiento}

El presente artículo de revisión no requirió financiación.

\section{Conflicto de intereses}

El autor declara no tener conflicto de intereses.

\section{Referencias bibliográficas}

1. Etiquetado de alimentos [Internet]. Organización de las Naciones Unidas para la Alimentación y la Agricultura. (Consultado el 22 de noviembre de 2020). Disponible en: http://www.fao.org/food-labelling/es/

2. Etiquetado nutricional frontal de alimentos [Internet]. Ministerio de Salud y Desarrollo Social. 2018. (Consultado el 29 de noviembre de 2020]. Disponible en: https://www. fbioyf.unr.edu.ar/evirtual/pluginfile.php/174457/mod_ resource/content/2/INFORME-etiquedato-nutricionalfrontal-alimentos\%202018.pdf

3. Aschemann-Witzel J, Grunert KG, van Trijp HCM, Bialkova S, Raats MM, Hodgkins C, et al. Effects of nutrition label format and product assortment on the healthfulness of food choice. Appetite. 2013;71:63-74. doi: 10.1016/j.appet.2013.07.004.

4. Plan de acción para la prevención de la obesidad en la niñez y la adolescencia [Internet]. Organización Panamericana de la Salud. 2015. (Consultado el 29 de noviembre de 2020). Disponible en: https://www.paho.org/hq/dmdocuments/2015/obesity-plan-of-action-child-spa-2015.pdf

5. Cabrera JA. Estudio sobre la influencia del etiquetado frontal en los alimentos sobre el comportamiento de los consumidores en diversos países y en Colombia. Breve revisión del estado actual. Rev Alimentos Hoy. 2020;28(49):2020.

6. Revisión sobre etiquetado frontal de alimentos y sistemas de perfiles nutricionales en el marco del diseño de políticas públicas [Internet]. Centro de Estudios sobre Políticas y Economía de la Alimentación. (Consultado el 22 de noviembre de 2020). Disponible en: https://cepea.com.ar/cepea/wpcontent/uploads/2018/06/Revision-etiquetado-y-perfilesjunio-2018-versi\%C3\%B3n-final.pdf

7. Colombia tendrá etiquetado nutricional en los alimentos envasados [Internet]. MinSalud. (Consultado el 29 de noviembre de 2020). Disponible en: https://www.minsalud. gov.co/Paginas/Colombia-tendra-etiquetado-nutricional-enlos-alimentos-envasados.aspx 
8. Santos G, Bravo-Rebatta F, Velarde Delgado P, Aramburu A. Efectos del etiquetado nutricional frontal de alimentos y bebidas: sinopsis de revisiones sistemáticas. Rev Panam Salud Pública. 2019;43:1. doi: 10.26633/RPSP.2019.62.

9. Setareh S, Jahn S, Boztug Y. Dual-process theory and consumer response to front-of-package nutrition label formats. Nutr Rev. 2017;75(11):871-82. doi: 10.1093/nutrit/nux043.

10. Dorantes D, Naranjo S. Etiquetado frontal: entre la mercadotecnia y las políticas de salud pública. Nutr Clín Diet Hosp. 2011;31(3):52-61.

11. Arrúa A, Curutchet MR, Rey N, Barreto P, Golovchenko N, Sellanes A, et al. Impact of front-of-pack nutrition information and label design on children's choice of two snack foods: Comparison of warnings and the traffic-light system. Appetite. 2017;116:139-46. doi: 10.1016/j.appet.2017.04.012.

12. Ares G, Varela F, Machin L, Antúnez L, Giménez A, Curutchet $\mathrm{MR}$, et al. Comparative performance of three interpretative front-of-pack nutrition labelling schemes: Insights for policy making. Food Qual Prefer. 2018;68:215-25. Doi: 10.1016/j. foodqual.2018.03.007.

13. Khandpur N, de Morais Sato P, Mais LA, Bortoletto Martins AP, Spinillo CG, Garcia MT, et al. Are front-of-package warning labels more effective at communicating nutrition information than traffic-light labels? A randomized controlled experiment in a Brazilian sample. Nutrients. 2018;10(6). doi: 10.3390/nu10060688.

14. Ley 20606, sobre composición nutricional de los alimentos y su publicidad [Internet]. Ministerio De Salud, Subsecretaría De Salud. (Consultado el 2 de diciembre de 2020]. Disponible en: https://www.bcn.cl/leychile/navegar?idNorma $=1041570$

15. A tres años de Ley Etiquetado: cambio en la composición de productos y en los hábitos de compra, [Internet]. Instituto Transparente. (Consultado el 2 de diciembre de 2020). Disponible en: https://inta.cl/a-tres-anos-de-ley-etiquetadocambio-en-la-composicion-de-productos-y-en-los-habitosde-compra/

16. Sebastián-Ponce MI, Sanz-Valero J, Wanden-Berghe C. Información percibida por los consumidores a través del etiquetado sobre las grasas presentes en los alimentos: revisión sistemática. Nutr Hosp.2015;31(1):129-42.

17. Coleman $\mathrm{CH}$, Bouësseau $\mathrm{MC}$, Reis A. Contribución de la ética a la salud pública [Internet]. Boletín Organización Mundial Salud. 2008;86:577-656.

18. Schramm FR, Kottow M. Principios bioéticos en salud pública: limitaciones y propuestas. Cad Saúde Pública. 2001;17(4):949-56.

19. Cárdenas D, Bermúdez C, Echeverri S, Pérez A, Puentes M, López L, et al. Declaración de Cartagena. Declaración Internacional sobre el Derecho al Cuidado Nutricional y la Lucha contra la Malnutrición. Nutr Hosp. 2019;36(4):97480. doi: $10.20960 /$ nh.02701.
20. Nutrición y etiquetado [Internet]. Organización de las Naciones Unidas para la Alimentación y la Agricultura. (Consultado el 2 de diciembre de 2020). Disponible en: http://www.fao.org/fao-who-codexalimentarius/themes/ nutrition-labelling/es/

21. Huerta A, Morán Rey F. Aplicación del etiquetado frontal como medida de salud pública y fuente de información nutricional. Rev Esp Nutr Comunitaria. 2015; (2):34-42. doi: 10.14642/RENC.2015.21.2.5101.

22. Información alimentaria: cuestiones éticas y jurídico políticas [Internet]. Cátedra Unesco de Bioetica; Observatorio de Bioética y Derecho de la Universidad de Barcelona. (Consultado el 2 de diciembre de 2020). Disponible en: http://www.bioeticayderecho.ub.edu/es/informacion-alimentaria-cuestiones-eticas-y-juridico-politicas

23. Álvarez Mejía SP.El principio de corresponsabilidad en salud en colombia. [Internet]. Tesis demaestríay doctorado. (Consultado el 2 de diciembre de 2020). Disponible en: https://repository.upb.edu.co/bitstream/handle/20.500.11912/2626/ ART\%C3\% 83\%20CULO \%20Sandra\%20Patricia \%20 $\%$ C3\%83\%20lvarez\%20Mejia.pdf?sequence=1

24. Anzola Nieves A. La corresponsabilidad como principio constitucional en Venezuela. Cuest Const. 2012;(26):3-29.

25. De Luise L, Dueñas S. Etiquetado frontal de alimentos: antecedentes y situación actual [Internet]. Consultado el 2 de diciembre de 2020). Disponible en: https://docplayer. es/198191355-Toda-persona-tiene-derecho-a-la-informacion-de-lo-que-consume-en-un-alimento-procesado.html

26. A dos años de implementación, cae aprobación de etiquetado [Internet]. La Tercera. 2018. (Consultado el 1 de marzo de 2020). Disponible en: https://www.latercera.com/nacional/noticia/ dos-anos-implementacion-cae-aprobacion-etiquetado/221634/

27. Torralba JM. La teoría kantiana de la acción: de la noción de máxima como regla autoimpuesta a la descripción de la acción. Tópicos México. 2011;(41):17-61.

28. Kant E. Fundamentación de la metafísica de las costumbres. Puerto Rico: Ed. Pedro M Rosario Barbosa. 2007.

29. Sellés JF, Burgos JM. Antropología: una guía para la existencia. Madrid: Palabra. 2003. p. 423.

30. Determinantes sociales de la salud [Internet]. Organización Mundial de la Salud. (Consultado el 2 de diciembre de 2020). Disponible en: https://www.who.int/social_determinants/es/

31. Mújica OJ. Cuatro cuestiones axiológicas de la epidemiología social para el monitoreo de la desigualdad en salud. Rev Panam Salud Pública. 2015;38:433-41. doi: 10665.2/18557/ v38n6a1_433-441.

32. Capdevielle J. El concepto de habitus: «con Bordieu y contra Bordieu». Anduli Rev Andal Cienc Soc. 2011;(10):31-46. doi: $10.12795 /$ anduli. 